\title{
A cyclodextrin-based approach for selective detection of catecholamine hormone mixtures
}

\author{
Jung-Hoon Yang, Hyun Tae Kim and Hanseup Kim ${ }^{*}$
}

\begin{abstract}
This paper presents an electrochemical sensing approach that enables quantitative detection of three major catecholamine hormones from a mixture by specifically employing a chemically-modified microelectrode array with $a-, \beta$ - and $\gamma$-cyclodextrin (CD) 'catchers' holding unique physical matching (size and shape) as well as chemical enticing (stereochemistry and surface charge) properties. The developed neurotransmitter sensor has selectively identified L-tyrosine, dihydroxyphenylalanine (L-DOPA) and dopamine in the absence of ascorbic acid. It exhibited the relatively linear sensitivities to each neurotransmitter with logarithmically increasing concentrations range of $5 \mu \mathrm{M}-10 \mathrm{mM}$, while demonstrating stability up to 6 hours from the fabrication and the average accuracy of 91.2\%.
\end{abstract}

Keywords: Cyclodextrins; Catecholamine hormone; Neurotransmitter; Micro electrochemical sensor

\section{Introduction}

Neurodegenerative diseases present an increasing burden for the world health care sector. Currently, 6.5 million Americans suffer from the neurodegenerative diseases such as Alzheimer's disease (5.4 million), Parkinson's disease (1 million), amyotrophic lateral sclerosis $(30,000)$, and Huntington's disease $(15,000)$ [1-3]. Since the neurodegenerative diseases are primarily an age-based disease with the increasing incidence after age 65 years $[4,5]$ and the U.S. population continue to age [6], it is estimated that, by 2040, more than 13.1 million Americans will be diagnosed with neurodegenerative diseases. Such increase of the neurodegenerative disease population will cause the corresponding growth of social costs of treating neurodegenerative diseases; thus, developing effective treatments for the neurodegenerative diseases is an urgent and critical issue.

For diagnosing and treating the neurodegenerative diseases, monitoring the concentrations of co-existing catecholamine neurotransmitters could be critical because concentrations represent the effectiveness of the derangement of neurotransmitters transferring through a neuron terminal and was shown to be associated with many neurodegenerative diseases. For example, Parkinson's disease has high correlation with the lack of dopamine (DA) due to the

\footnotetext{
* Correspondence: hanseup@ece.utah.edu

Electrical and Computer Engineering, University of Utah, 36 S. Wasatch drive, 84112 Salt Lake City, UT, USA
}

loss of dopaminergic neurons in the substantia nigrapars compacta and degeneration of the nigrostriatal pathway [7-9]. Although DA concentration is the direct cause of such diseases, since the biosynthetic pathway of major catecholamine neurotransmitters has been well-understood (Figure 1), the concentration measurements of these individual neurotransmitters (L-Tyrosine, L-DOPA and DA) could provide meaningful insights on the failure mechanisms along the pathway. L-tyrosine is converted into L-DOPA by Tyrosine hydroxylase enzyme, while L-DOPA, in turn, is transformed into DA through Dopa decarboxylase enzyme. Thus, the extra-ordinarily low concentration of DA while normal concentrations of L-tyrosine and L-DOPA would indicate the damage of Dopa decarboxylase enzymes or its neuronal pathway. Such insights, thus, could be an excellent indicator of the status of some neurodegenerative diseases. Therefore, the monitoring of DA as well as the closest pre-derivatives of dopamine, such as L-tyrosine and L-DOPA, would be the main focus of this study.

The detection of neurotransmitters has been performed largely in four ways: electrochemical detection (ECD) [10-17], fluorescence detection (FD) [18-21], chemiluminescence detection (CLD) [22-24], and mass spectrometric detection (MSD) [25]. However, none of those approaches have been successful in selectively detecting the co-existing individual catecholamine neurotransmitters without using additional separation techniques such as liquid chromatography or capillary electrophoresis that require complex 


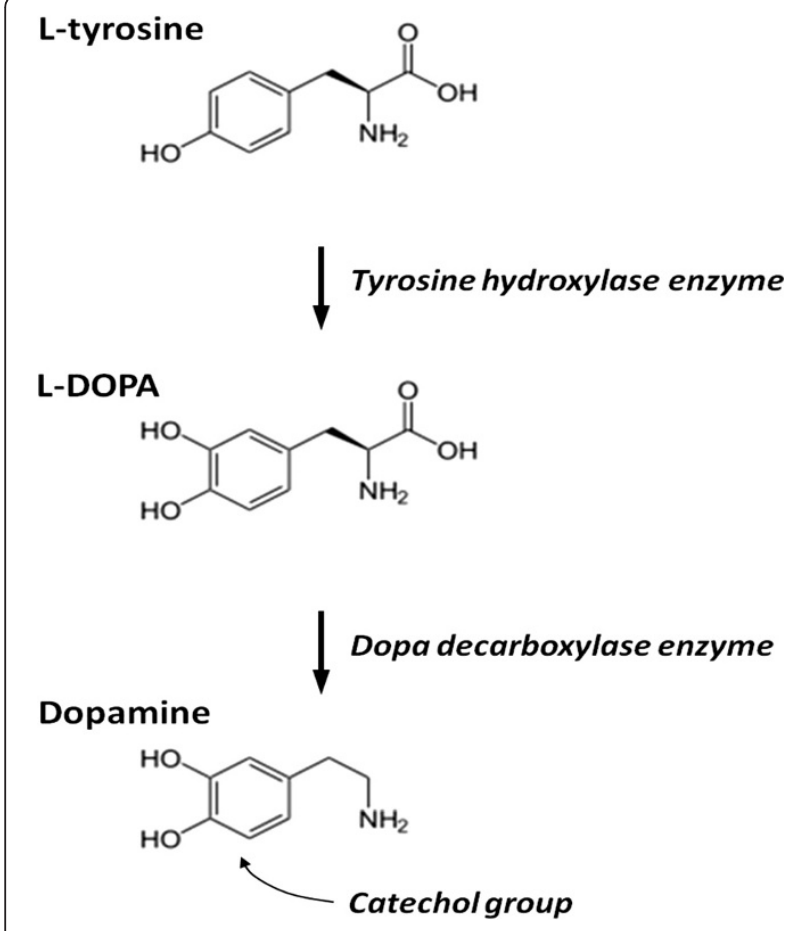

Figure 1 Biosynthetic pathway of catecholamine neurotransmitters.

system, long analysis time, and high power consumption. Particularly, the ECD has been most frequently utilized for the neurotransmitter detection due to its advantages of high sensitivity, real time analysis, low cost, and easiness of system miniaturization $[17,26]$; however, only the detection of a single catecholamine neurotransmitter has been successfully achieved because the conventional enzyme-based electrochemical sensors, based on the redox reaction of catechol groups with tyrosinase, was incapable of distinguishing the neurotransmitters because each DA and L-DOPA has identical catechol groups (two $\mathrm{OH}$ groups attached to one benzene ring) that similarly react with the enzyme attached to electrodes and generate redox reaction current.

To overcome such limitation we developed an electrochemical sensor using a differently-chemically-modified microelectrode array with various CycloDextrin (CD) 'catchers' that are capable of engaging differently-sized target molecules respectively due to physically unique molecular structures. Note that previously it was known that a CD could trap certain molecules better due to its "basket"-type physical shapes, also known as the host-guest recognition [12-14,27-30]; however, it has not been applied for the selective identification of multiple catecholamine neurotransmitters. Here we focus on the use of the CDs' size differences to statistically produce unique affinities to each of co-existing catecholamine neurotransmitters. Especially, we employed three different $\alpha-, \beta-$, and $\gamma$-CD catchers, which have respectively six, seven, and eight D-glucopyranose units based on their physically 'matching' sizes (Figure 2), to identify three targets of L-tyrosine, L-DOPA and DA.

Recently the use of cyclodextrins has been proven to detect dopamine (DA) despite the interference of ascorbic acid (AA) in a mixture [31-33]. Ascorbic acid has been known to interfere the detection of dopamine due to the overlap of the oxidation potentials and much higher $(>100$ times) concentrations. Based on these previous results, it was reasonably hypothesized that the proposed approach would properly function even in the presence of ascorbic acids. Thus, in this paper we report the simultaneous and quantitative detection approach to distinguish L-tyrosine, L-DOPA and Dopamine in the absence of ascorbic acids as the proof-of-concept. Particularly, we report the structure, fabrication and characterization of a micro neurotransmitter sensor as the proof-of-concept device, with particular foci on detecting DA as well as its closest pre-derivatives. The details will be reported of (1) microelectrode array fabrication, (2) electrode functionalization, (3) sensing sensitivity and selectivity and (4) simultaneous identification of the mixture ratios among L-tyrosine, L-DOPA and Dopamine.

\section{Findings}

\section{Structure and operation principle}

The developed micro neurotransmitter sensor consists of a microelectrode array and an electrochemical chamber on a silicon substrate (Figure 3-right bottom). The microelectrode array has four working electrodes $(2 \mathrm{~mm}$ diameter $)$ at which electrochemical reaction takes place and one counter electrode at the center among the four working electrodes. Three of the four working electrodes are differently and chemically functionalized by immobilizing $\alpha-, \beta-$, and $\gamma$-CDs respectively on its surface, while the fourth electrode is not coated as a reference electrode. The counter electrode works as a ground in potential measurement. The electrochemical chamber contains the target solution with neurotransmitters and exposes it to all five electrodes for analysis. The total footprint of the sensor was $13 \times 21 \mathrm{~mm}^{2}$.

The developed neurotransmitter sensor produces three output signals through the host-guest recognition from each of the three CD-coated electrodes, and the combination of those three signals enables the identification of each concentration of the co-existing three target neurotransmitters, such as L-tyrosine, L-DOPA and dopamine. Since each electrode is immobilized with different CDs with unique size, shape, stereochemistry and surface charge, its surface demonstrates variable affinities to each catecholamine neurotransmitter. For example, the total output voltage, measured at the $\alpha-C D$ electrode, is the combined results from the respective responses to L-tyrosine, L-DOPA and dopamine. Thus, each output signal at each electrode is the combined contribution of each CD to all three target neurotransmitters. By combining such a relationship from all 


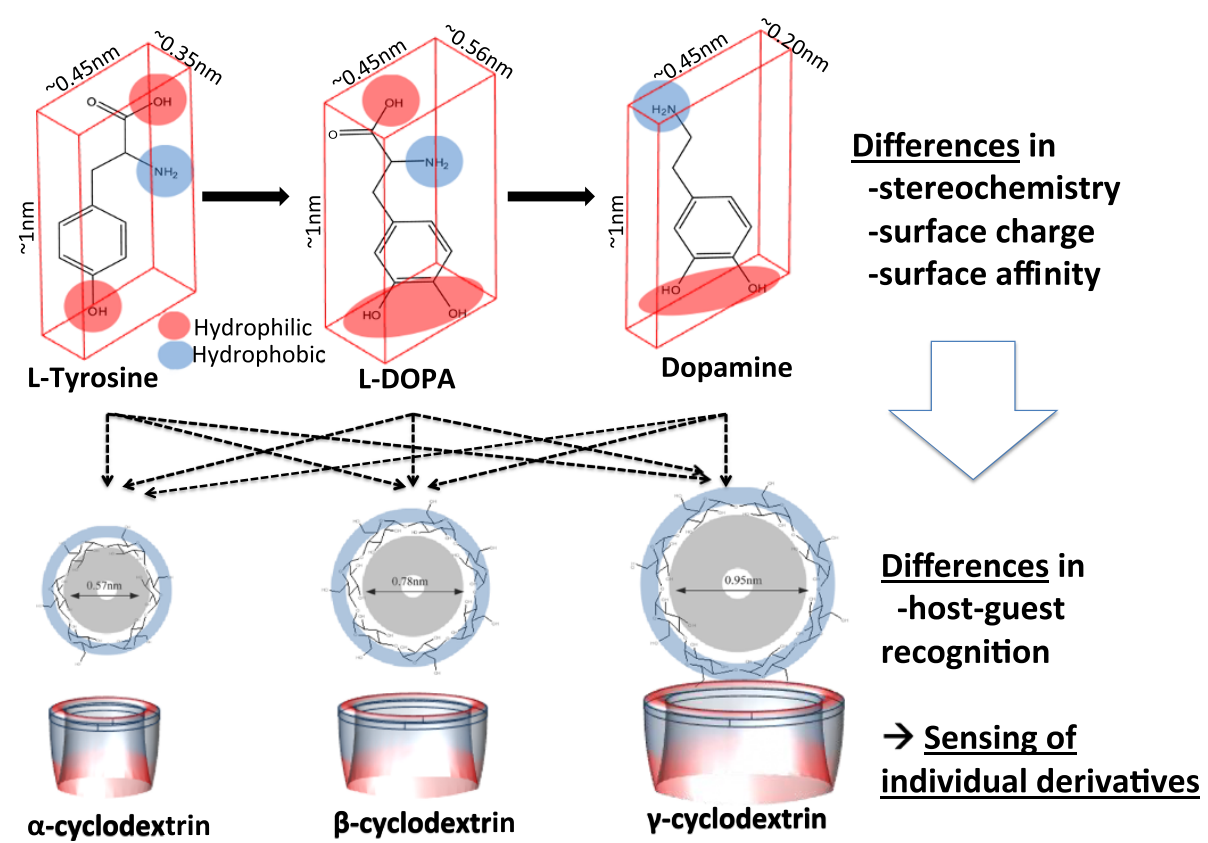

Figure 2 Illustration of the sensing principle: differences in stereochemistry, surface charge and surface affinity of the three catecholamine neurotransmitter derivatives cause different levels of host-guest recognition to $\alpha-, \beta$ - and $\gamma$-cyclodextrins, respectively, enabling the sensing of individual derivative ratios from a mixture.

three electrodes coated with three $\alpha-, \beta-$, and $\gamma^{-C D s}$, the relationship of the output signals can be expanded into three equations:

$$
\begin{aligned}
& A=\alpha_{1} \cdot L T+\alpha_{2} \cdot L D+\alpha_{3} \cdot D A+\alpha_{4} \\
& B=\beta_{1} \cdot L T+\beta_{2} \cdot L D+\beta_{3} \cdot D A+\beta_{4} \\
& C=\gamma_{1} \cdot L T+\gamma_{2} \cdot L D+\gamma_{3} \cdot D A+\gamma_{4}
\end{aligned}
$$

where $\mathrm{A}, \mathrm{B}$ and $\mathrm{C}$ are the measured output signals at each electrode with $\alpha$-, $\beta$ - and $\gamma$-cyclodextrins (CDs) coating, respectively. $\alpha_{1}, \alpha_{2}$, and $\alpha_{3}$ are the response coefficients of the $\alpha-C D$ electrode, while $\alpha_{4}$ are the sum of the offset values to each target neurotransmitter. The same relationship exists for $\beta_{1}, \beta_{2}, \beta_{3}, \beta_{4}, \gamma_{1}, \gamma_{2}, \gamma_{3}$ and $\gamma_{4}$. LT, LD and DA indicate the concentrations of L-tyrosine, L-DOPA and DA, respectively. Under the hypothesis that this matrix is consistent (i.e. all the coefficients are independent), this set of three equations could be solved to determine the concentrations of LT, LD, and DA simultaneously, therefore enabling the identification of the co-existing three neurotransmitters of interests.

$$
\left[\begin{array}{l}
L T \\
L D \\
D A
\end{array}\right]=\left[\begin{array}{lll}
\alpha_{1} & \alpha_{2} & \alpha_{3} \\
\beta_{1} & \beta_{2} & \beta_{3} \\
\gamma_{1} & \gamma_{2} & \gamma_{3}
\end{array}\right]^{-1} \cdot\left[\begin{array}{l}
A-\alpha_{4} \\
B-\beta_{4} \\
C-\gamma_{4}
\end{array}\right]
$$

Note that in order to prove the selectivity of the method, one exemplary ascorbic acid (vitamin C) was mixed into a solution for in-vivo-like consideration while measuring the three neurotransmitters.

\section{Fabrication}

\section{Micro electrode array fabrication}

The microelectrode array was fabricated on a silicon substrate (Figure 3-left). First, a $\mathrm{SiO}_{2}$ layer $(200 \mathrm{~nm}$ ) was grown by wet oxidation, which served as an insulating layer. Second, a TiW/Au layer $(30 \mathrm{~nm} / 600 \mathrm{~nm}$ ) was sputtered on top of the $\mathrm{SiO}_{2}$ layer, where the TiW layer enhanced the adhesion between the $\mathrm{Au}$ and $\mathrm{SiO}_{2}$. Third, the deposited metal layers were patterned using photolithography. KI and diluted $\mathrm{H}_{2} \mathrm{O}_{2}$ solution were used as metal etchant and Shipley 1813 positive photoresist as an etch mask. Finally, the patterned substrate was cleaned by Acetone, IPA, and DI water. The footprint of each electrode was $7.065 \mathrm{~mm}^{2}$.

\section{Electrode functionalization}

The fabricated microelectrode array was functionalized by immobilizing CDs on its surface (Figure 3-right top). First, the fabricated device was soft-baked on a hot plate at $80^{\circ} \mathrm{C}$ for $10 \mathrm{~min}$ to remove residual water molecules on the electrode surface because water molecules can prevent uniform coating of CDs molecules. Second, it was treated in a $1 \mathrm{mM}$ cysteamine for $12 \mathrm{hr}$, which was prepared in ethanol, for functionalizing amino-terminated monolayer. Third, the modified electrode array was immersed in a $30 \mathrm{mM}$ of chlorobutyric acid (CA) for $2 \mathrm{hr}$, which was mixed with $0.1 \mathrm{M} \mathrm{N}$-hydroxysuccinimide (NHS) and $0.4 \mathrm{M}$ ethyl (dimethylaminopropyl) carbodiimide (EDC). This procedure enabled to form amide binding between the chlorobutyric acid and amino group, which changed the functionalized 


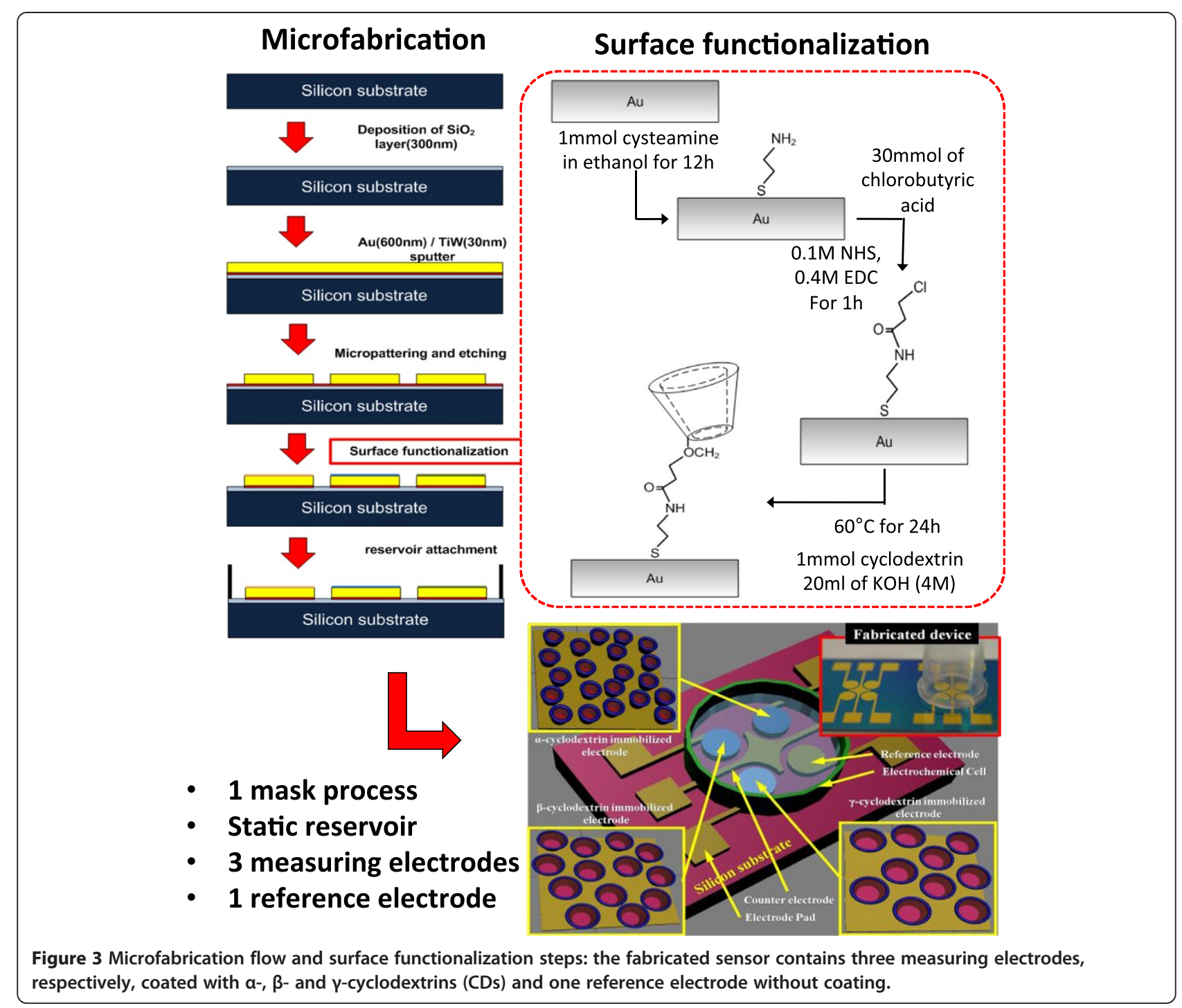

electrode to be hydrophilic. Finally, small droplets of the each mixture of $1 \mathrm{mM} \alpha-, \beta-$, and $\gamma$-CDs and $20 \mathrm{ml}$ of aqueous $4 \mathrm{M} \mathrm{KOH}$ were selectively deposited on top of each arrayed electrode at $65^{\circ} \mathrm{C}$ for $24 \mathrm{hr}$. The $\alpha$-, $\beta$-, and $\gamma$-CDs and all the chemicals that used for CD immobilization such as Cysteamine (95\%), CA, NHS, and EDC were purchased from Sigma-Aldrich Co., USA.

\section{Packaging}

The functionalized microelectrode array was connected to an electrochemical measurement system using copper wire. The room temperature curable conductive paste (Silver conductive adhesive paste, Alfa Aesar) was used to bond the wire such that the immobilized CDs could avoid the thermal damage from conventional solder bonding. While curing the conductive paste, the microelectrode array was covered with a water-absorbed filter paper (1002-185, Whatman) to keep the CDs being sufficiently moisturized. After the conductive paste cured, an electrochemical chamber was built on top of the fabricated sensor chip by bonding a polycarbonate tube (inner diameter $10 \mathrm{~mm}$, length $15 \mathrm{~mm}$ ) with epoxy (Quick Set ${ }^{\mathrm{TM}}$, Loctite).

\section{Methodology}

\section{Analysis of the functionalized electrode}

The functionalized microelectrode array was analyzed utilizing Fourier transform infrared spectrometry (FT-IR) and X-ray photoelectron spectroscopy (XPS) to confirm the immobilization of $\alpha-, \beta$-, and $\gamma$-CDs. The FT-IR results identify existence of chemical bindings after each step of electrode functionalization over a wavelength range of 500-4000 nm (Perkin-Elmer Spectrum 100), while the XPS measurements identify the existing atoms on the electrode over electron beam power of 0-1000 eV (Kratos analytical Axis Ultra DLD). 


\section{Electrochemical measurement}

The fabricated neurotransmitter sensor was connected to a potentiostat (Reference 600, Gamry Instruments) and test data was monitored using a labview-based data recording system (VFP600). The electrochemical cell was filled with target catecholamine hormone solution, and the fabricated sensor was tested in random mixtures of catecholamine hormone targets (L-tyrosine, L-DOPA, and DA), concentrations $(5 \mu \mathrm{M}-10 \mathrm{mM})$, and scan rate $(1-100 \mathrm{mV} / \mathrm{s})$. First, $\mathrm{I}-\mathrm{V}$ curves of each electrode were measured in a specific neurotransmitter concentration to measure the individual responses of each CDs immobilized electrode. Next, by varying the neurotransmitter concentrations between $5 \mu \mathrm{M}-10 \mathrm{mM}$ at every decade, the response curves were constructed for each $\mathrm{CD}$ electrode and neurotransmitter. The slope of the curve provides the sensitivity of each electrode to each neurotransmitter. Then, from the collected sensitivity coefficients, the matrix of quantification was constructed to identify the individual concentrations from a mixture. Finally, the individual concentrations were extracted from random mixtures of L-tyrosine, L-DOPA and dopamine and compared to the original values, in order to validate the developed sensor and method. Measurement was repeated at least five times, and all the catecholamine hormones were purchased from SigmaAldrich Co., USA.

\section{Results and discussion}

\section{Electrode functionalization}

FT-IR spectra measurements at each stage of the surface coating indicated that the CDs were successfully immobilized on the Au surface of each electrode, as shown in Figure 4-top. First, the characteristic peaks of $-\mathrm{NH}_{2}$ bending vibration and the symmetrical stretching vibration of $\mathrm{C}-\mathrm{N}$ appeared at $1545 \mathrm{~cm}^{-1}$ and at $1153 \mathrm{~cm}^{-1}$, respectively after the first step of cystamine treatment on Au electrodes. This indicates the formation of first amine functional layer for the subsequent hydrogen bonding. Second, two new peaks were measured at $1018 \mathrm{~cm}^{-1}$ and $1732 \mathrm{~cm}^{-1}$ after the treatment of 4-chlorobutyric acid on the Au electrode. These peaks correspond to the $\mathrm{C}-\mathrm{O}-\mathrm{C}$ stretching vibration and amide binding between amine and carboxylic group, respectively, indicating that the outermost coating now becomes chlorobutyric acid available for CD immobilization. Finally, the additional new peaks were also observed at $847 \mathrm{~cm}^{-1}$ (C-H deformation in $\alpha$-pyranose compounds) and $953 \mathrm{~cm}^{-1}$ (asymmetric pyranose ring stretch). These peaks are conventional signatures for typical CDs, evidencing that CDs had been immobilized on the Au surface as the outmost chemical acceptor.

XPS survey scan measurements, complementing the FT-IR spectra results, indicated that the key elements, such as nitrogen and chlorine, appeared and disappeared as the surface coating progresses. Figure 4-bottom displays

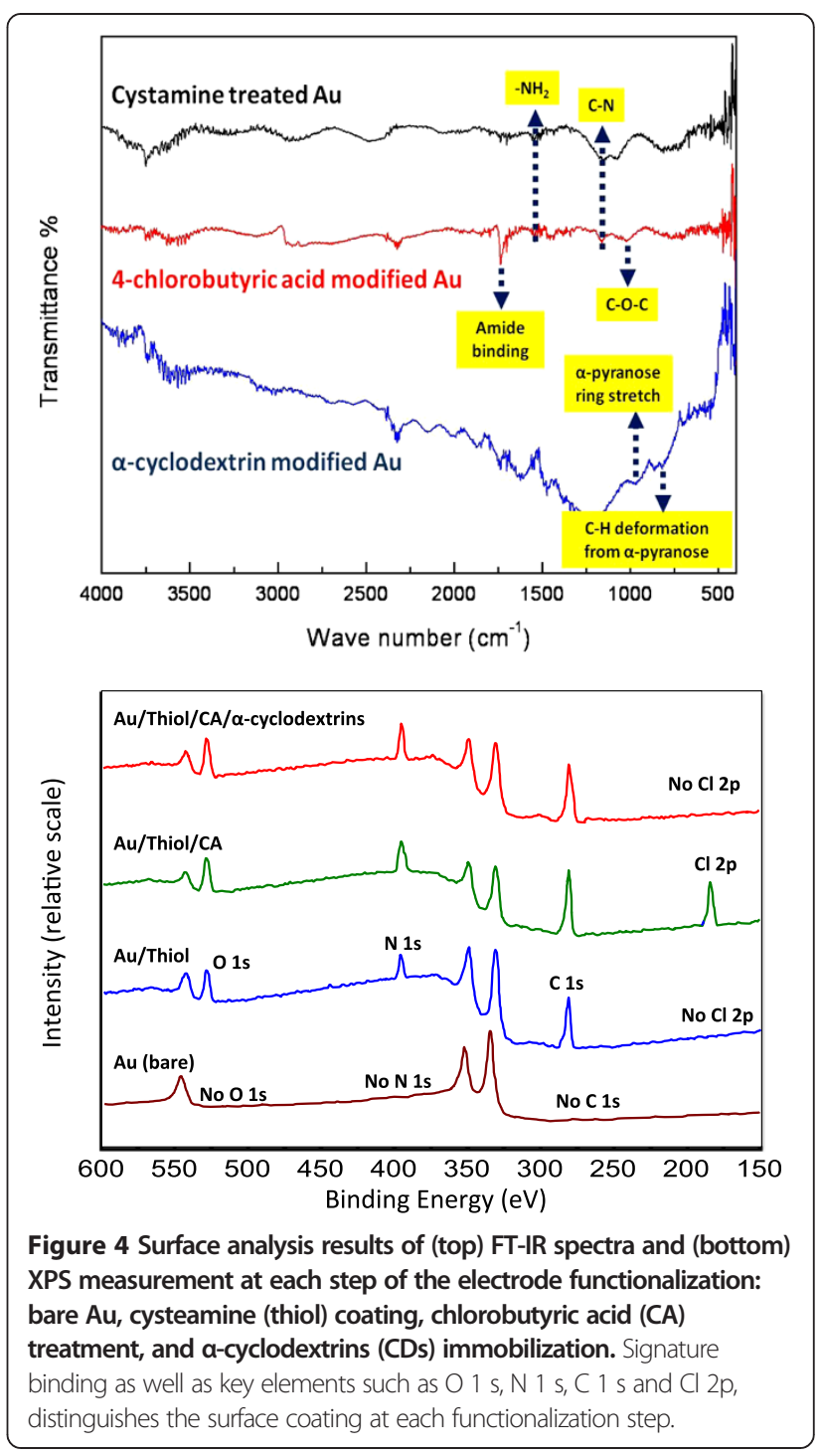

the baseline binding energy of each element at the bare $\mathrm{Au}$ surface. In comparison, the peak of nitrogen ( $\mathrm{N} 1 \mathrm{~s}$ ) was observed at the binding energy of $397.1 \mathrm{eV}$ after cysteamine treatment that creates amino termination. The peak for carbon ( $\mathrm{C} 1 \mathrm{~s}$ ) was also measured throughout the coating steps at the binding energy of $281.6 \mathrm{eV}$. Then, an additional peak of chlorine $(\mathrm{Cl} 2 \mathrm{p})$ was measured at the binding energy at $192.6 \mathrm{eV}$, after chlorobutyric acid treatment that forms chlorine termination. Note that the oxygen ( $\mathrm{O} 1 \mathrm{~s})$ spectrum was observed as a distinctive peak at $529.4 \mathrm{eV}$, indicating the increased hydrophilicity on the functionalized Au surface. This enables the surface to easily interact with water molecules thus efficiently capture CDs. The peak of chlorine was not measured once the cholorobutyric acid replaced chlorine element with $\alpha$-CDs. This confirms the existence of the $\alpha$-CDs on the Au electrode. 


\section{Identification of each catecholamine neurotransmitter from a mixture}

Cyclic voltammetry measurements demonstrated that each electrode responses to respective neurotransmitter with different order of preferences and degrees (Figure 5). The $\gamma$-electrode produced the highest output under the same concentration of L-tyrosine, while the $\beta$-electrode showed the maximum signal outputs from both L-DOPA and dopamine. Although the $\alpha$-electrode did not demonstrate the highest output to any of the neurotransmitters, it responded the second highest output to L-tyrosine and dopamine. Figure 5 shows the cyclic voltammograms respectively obtained from $1 \mathrm{mM}$ of L-tyrosine, L-DOPA and DA in PBS from each electrode with the footprint of $7.065 \mathrm{~mm}^{2}$, resulting in different output responses under the same neurotransmitter. In response to $1 \mathrm{mM}$ of L-tyrosine at the L-tyrosine oxidation potential, the $\gamma$-electrode produced the highest output current of $1.765 \mu \mathrm{A}$ and then the $\beta$ - and $\alpha$-electrodes produced 1.176 and $0.515 \mu \mathrm{A}$, respectively (Figure 5-top). This corresponds to the current densities of $0.250,0.167$ and $0.073 \mu \mathrm{A} / \mathrm{mm}^{2}$. The electrodes with chlorobutyric acid or cysteamine coating without cyclodextrins did not produce measurable output currents. The oxidation potential of each neurotransmitter was separately obtained utilizing conventional platinum electrodes. In response to LDOPA, the generated output signals ranged at 12.123, 7.397 and $6.37 \mu \mathrm{A}$ with coatings of $\beta-, \gamma^{-}, \alpha$-cyclodextrins, chlorobutyric acid and cystemine (Figure 5-Middle), with corresponding current densities of 1.716, 1.047 and $0.902 \mu \mathrm{A} / \mathrm{mm}^{2}$, respectively. In the $1 \mathrm{mM}$ dopamine solution, the $\beta$-, $\alpha$ - and $\gamma$-electrodes produced current outputs of $11.811,9.213$ and $7.323 \mu \mathrm{A}$ and densities of $1.672,1.304$ and $1.037 \mu \mathrm{A} / \mathrm{mm}^{2}$, respectively (Figure 5-bottom). These results indicate that each cyclodextrins, thus each electrode, holds different affinity in catecholamine hormones. Note that the minimum detectable amount was $5 \mu \mathrm{M}$, limited by the resolution of measurement. Also note that the testing was performed up to $10 \mathrm{mM}$ of concentrations to fully cover the maximum range feasible in-vivo environment. Thus, the overall concentration ranges tested were limited between $5 \mu \mathrm{M}$ and $10 \mathrm{mM}$ in this paper.

Amperometric measurement results demonstrated that the output current produced from each electrode with $\alpha-, \beta-$, and $\gamma$-immobilization increased linearly with increasing concentrations between 0.005 and $10 \mathrm{mM}$ in logarithmic scale (Figure 6). The linear relationship between each electrode and neurotransmitter produced substantially different slopes, which confirms selective discrimination of each electrode by the terminated CDs. In response to L-tyrosine, $\alpha-, \beta$ - and $\gamma$-electrodes respectively exhibited linear slopes of $0.2035,0.1055$ and 0.2897 , which forms a multiplication factor matrix as:
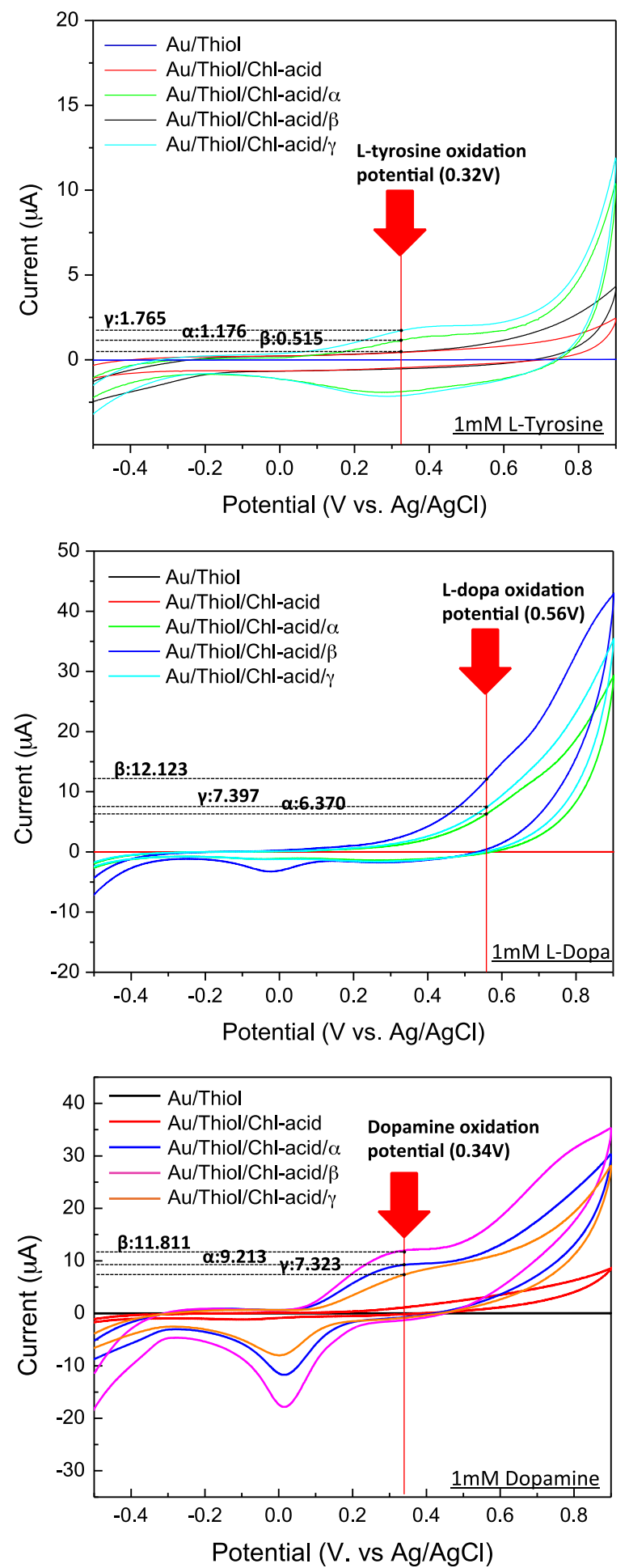

Figure 5 Cyclic voltammograms of each electrode with the $\alpha-, \beta-$, and $\gamma$-CDs immobilization, respectively, under the $1 \mathrm{mM}$ solutions of (top) L-tyrosine, (Middle) L-dopa and (bottom) dopamine. The scan rate was $100 \mathrm{mV} / \mathrm{s}$. The current amounts at the given concentration (1 $\mathrm{mM}$ ) were identified to produce the quantification matrix at each oxidation potential point that was measured without CD immobilization. The footprint of each electrode was $7.065 \mathrm{~mm}^{2}$. 


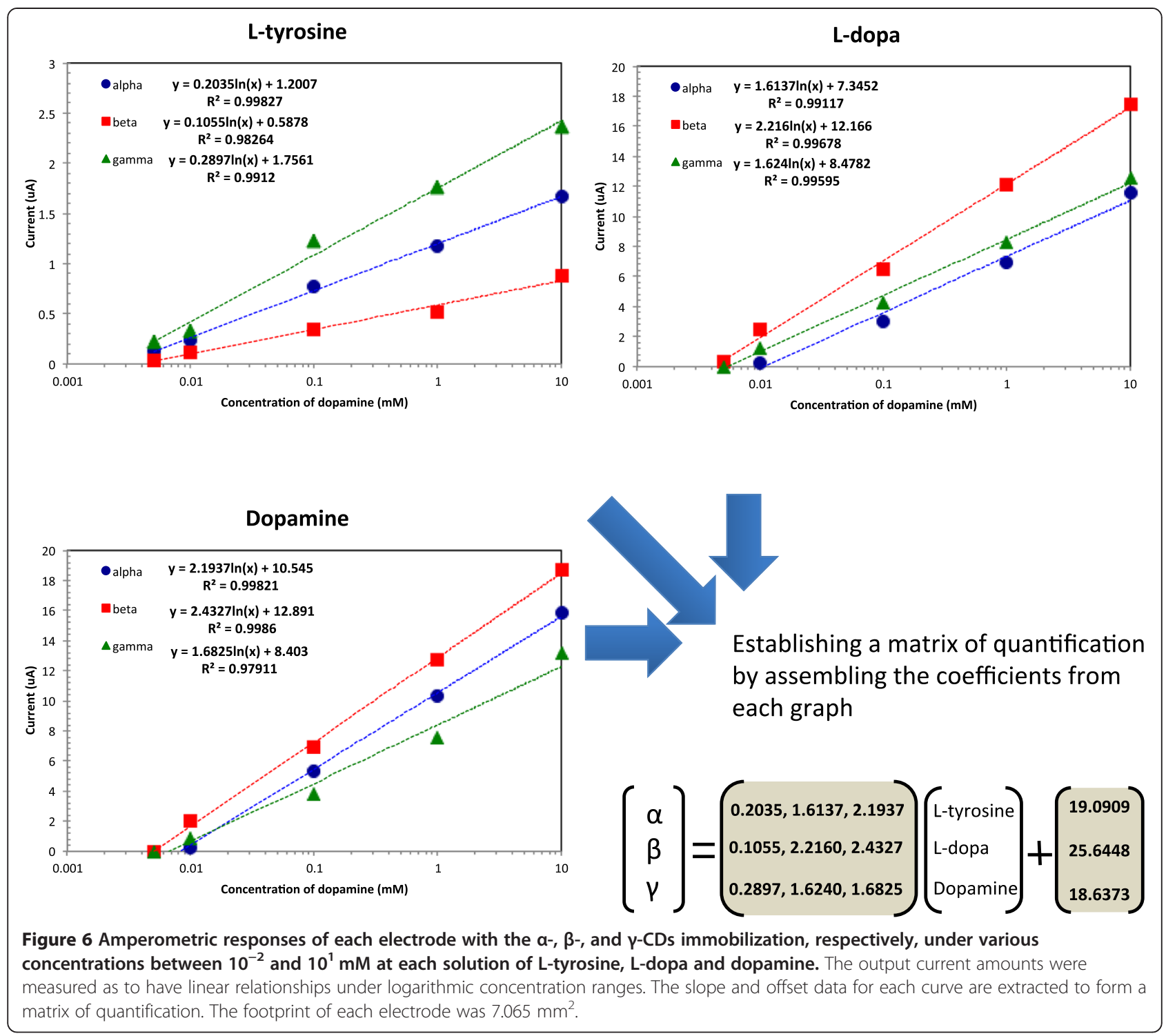

\section{Multiplication matrix for L-tyrosine

$$
=\left[\begin{array}{lll}
0.2035 & 0.1055 & 0.2897
\end{array}\right]
$$

By expanding the matrix to L-DOPA and dopamine, the complete output current can be expressed as:

$$
\begin{aligned}
& {\left[\begin{array}{l}
I(\alpha) \\
I(\beta) \\
I(\gamma)
\end{array}\right]=\left[\begin{array}{lll}
0.2039, & 0.1055, & 0.2897 \\
1.6137, & 2.2160, & 1.6240 \\
2.1937, & 2.4327, & 1.6825
\end{array}\right]\left[\begin{array}{l}
L T \\
L D \\
D A
\end{array}\right]} \\
& +\left[\begin{array}{l}
19.0909 \\
25.6448 \\
18.6373
\end{array}\right]
\end{aligned}
$$

where $I(\alpha), I(\beta)$ and $I(\gamma)$ are the output currents at each electrode and LT, LD and DA represent the concentrations in $\mathrm{mM}$. This matrix enables the detection of individual amounts of each neurotransmitters from a mixture.
Random mixture measurement results, without vitamin $\mathrm{C}$ (an ascorbic acid), demonstrated that individual concentrations of L-tyrosine, L-DOPA and dopamine can be identified utilizing the CD-based electrode as listed in Table 1. A total of three different mixture cases was evaluated at least five repeated experiments without ascorbic acids as explained earlier. The Mixture 1 included minute amounts of $0.035,0.010$ and $0.005 \mathrm{mM}$; the mixture 2 contained $0.39,1.11$, and $0.68 \mathrm{mM}$; and the Mixture 3 consisted of $1.10,3.24$ and $2.37 \mathrm{mM}$, respectively of L-tyrosine, L-DOPA and dopamine. Each mixture without ascorbic acids produced the output currents of 10.531, 8.921, and $11.531 \mu \mathrm{A} ; 18.722,18.724$ and $18.758 \mu \mathrm{A}$; and $20.73,21.11$, and $20.56 \mu \mathrm{A}$ respectively from $\alpha-, \beta$ - and $\gamma$-electrodes. By supplying the output currents into the constructed matrix of quantification, the supplied concentrations were estimated as $0.035,0.010$ and 
Table 1 Experimental measurement-based quantification results of three neurotransmitter mixture cases with and without ascorbic acid (1 $\mathrm{mM}$ of vitamin C): (Case I) 0.035, 0.010 and $0.005 \mathrm{mM}$; (Case II) 0.39, 1.11, and 0.68 $\mathrm{mM}$; and (Case III) 1.10, 3.24, and $2.37 \mathrm{mM}$ of L-tyrosine, L-dopa and dopamine, respectively

\begin{tabular}{|c|c|c|c|c|c|c|}
\hline \multicolumn{7}{|l|}{ Mixture I } \\
\hline \multirow{2}{*}{\multicolumn{2}{|c|}{ Input (No ascorbic acid) }} & \multicolumn{4}{|l|}{ Output } & \multirow[t]{3}{*}{ Accuracy output/input (error) } \\
\hline & & \multicolumn{2}{|c|}{ Measurement } & \multicolumn{2}{|c|}{ Estimated from measurement } & \\
\hline Type & $\mathrm{mM}$ & Electrode & $\mu \mathrm{A}$ & Type & $\mathrm{mM}$ & \\
\hline L-tyrosine & 0.035 & $a-C D$ & 10.531 & L-tyrosine & 0.0372 & $106.30 \%(+6.30 \%)$ \\
\hline L-dopa & 0.010 & $\beta-C D$ & 8.921 & L-dopa & 0.0093 & $92.83 \%(-7.17 \%)$ \\
\hline Dopamine & 0.005 & $\gamma-C D$ & 11.531 & Dopamine & 0.0052 & $105.05 \%(+5.05 \%)$ \\
\hline \multicolumn{7}{|l|}{ Mixture II } \\
\hline \multirow{2}{*}{\multicolumn{2}{|c|}{ Input (No ascorbic acid) }} & \multicolumn{4}{|l|}{ Output } & \multirow[t]{3}{*}{ Accuracy output/input (error) } \\
\hline & & \multicolumn{2}{|c|}{ Measurement } & \multicolumn{2}{|c|}{ Estimated from measurement } & \\
\hline Type & $\mathrm{mM}$ & Electrode & $\mu \mathrm{A}$ & Type & $\mathrm{mM}$ & \\
\hline L-tyrosine & 0.39 & $a-C D$ & 18.722 & L-tyrosine & 0.3915 & $100.37 \%(+0.37 \%)$ \\
\hline L-dopa & 1.11 & $\beta-C D$ & 18.724 & L-dopa & 1.1045 & $99.51 \%(-0.49 \%)$ \\
\hline Dopamine & 0.68 & $\gamma-C D$ & 18.758 & Dopamine & 0.6678 & $98.19 \%(-1.81 \%)$ \\
\hline \multicolumn{7}{|l|}{ Mixture III } \\
\hline \multirow{2}{*}{\multicolumn{2}{|c|}{ Input (No ascorbic acid) }} & \multicolumn{4}{|l|}{ Output } & \multirow[t]{2}{*}{ Accuracy output/input (error) } \\
\hline & & \multicolumn{2}{|c|}{ Measurement } & \multicolumn{2}{|c|}{ Estimated from measurement } & \\
\hline Type & $\mathrm{mM}$ & Electrode & $\mu \mathrm{A}$ & Type & $\mathrm{mM}$ & \\
\hline L-tyrosine & 1.10 & $a-C D$ & 20.73 & L-tyrosine & 1.1690 & $106.28 \%(+6.27 \%)$ \\
\hline L-dopa & 3.24 & $\beta-C D$ & 21.11 & L-dopa & 3.3399 & $103.08 \%(+3.08 \%)$ \\
\hline Dopamine & 2.37 & $\gamma-C D$ & 20.56 & Dopamine & 2.2724 & $95.88 \%(-4.12 \%)$ \\
\hline
\end{tabular}

Experimental data indicated above are the average of at least five experiments. The testing was performed within 10 minutes of fabrication.

\section{Accuracy vs Time}

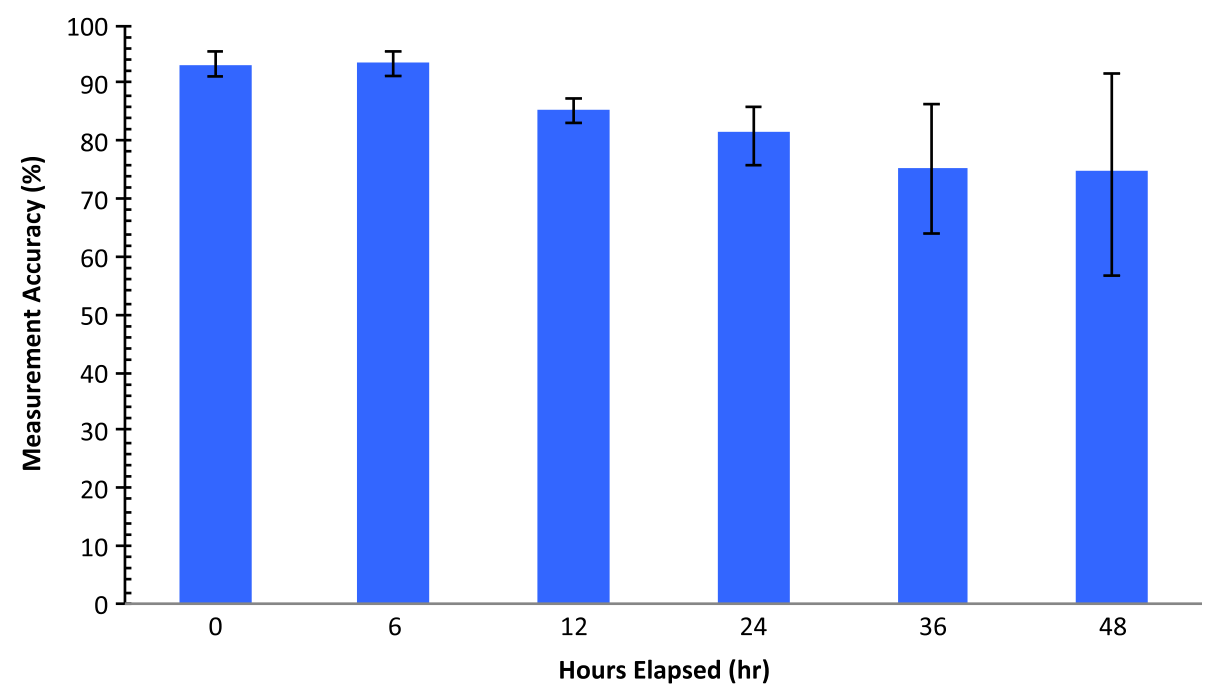

Figure 7 Composition identification accuracy over time utilizing $\alpha-, \beta-$, and $\gamma$-electrodes under 100uM ascorbic acid (vitamin C). Error bars indicate the deviation over five experiments. The results indicate that the neurotransmitter sensor provides stable outputs up to 6 hours. 
$0.005 \mathrm{mM}$; 0.39, 1.11 and $0.68 \mathrm{mM}$; and 1.10, 3.24 and $2.37 \mathrm{mM}$ respectively for the Mixtures 1, 2 and 3. These estimated results ranged within $92.41 \%$ and $106.30 \%$ of the supplied values for Mixtures 1, 2 and 3, verifying the concept of CD-based quantitative identification of mixed neurotransmitters. Note that all the Mixtures were evaluated within 6 hours of the device fabrication to minimize the performance drift over time.

The measurement results indicated that the average accuracy of the neurotransmitter sensor degraded over time from 93.2 (used immediately after fabrication) and 93.4 (within 6 hours) to 85.2, 81.4, 75.2 and $74.9 \%$, respectively for within 12, 24, 36 and 48 hours (Figure 7). The measurement also showed that the deviations of the accuracy increased over time from 2.1 to $18.2 \%$, indicating the reliable measurement periods after fabrication up to 6 hours.

\section{Conclusion}

A new neurotransmitter sensing technique has been examined to enables quantitative detection of three major catecholamine hormones from a mixture. Specifically, the sensing technique utilized three chemically-modified microelectrodes, respectively, with $\alpha$-, $\beta$ - and $\gamma$-cyclodextrin (CD) 'catchers'. The sensing technique relied on different physical matching (size and shape) as well as chemical enticing (stereochemistry and surface charge) properties of $\alpha-, \beta-$, and $\gamma$-CDs in order to produce statistically different affinities to each target catecholamine neurotransmitters of L-tyrosine, dihydroxyphenylalanine (L-DOPA) and dopamine. The developed neurotransmitter sensing technique has successfully identified three individual catecholamine hormones, without the existence of ascorbic acid, with respective $C D$ 'catchers': $\alpha$-CDs better responded to L-tyrosine and dopamine; $\beta$-CDs to L-DOPA and dopamine; and $\gamma$-CDs to L-tyrosine, respectively. It also demonstrated the linear sensitivities to each neurotransmitter with logarithmically increasing concentrations range of $5 \mu \mathrm{M}-10 \mathrm{mM}$. The measurement accuracy changed over time, indicating the stability up to 6 hours from the fabrication. The demonstrated accuracy, over $>5$ tests near $1 \mathrm{mM}$ of target concentrations, was within $91.2 \%$, and the total device footprint was $13 \times 21 \mathrm{~mm}^{2}$.

\section{Competing interests}

The authors declare that they have no competing interests.

\section{Authors' contributions}

JY performed the fabrication and testing of the developed electrochemical sensors. HTK performed a variety of electrochemical measurements. JY, HTK and HK analyzed the measurement data and drafted the manuscript. All authors read and approved the final manuscript.

\section{Authors' information}

Jung Hoon Yang received the B.E. degree in chemical engineering from Kyounggi University, Suwon, Korea, in 2001, the M.S. degree in bio-microsystem technology from Korea University, Seoul, Korea, in 2003, and the Ph.D. degree in nano science and engineering from Waseda University, Tokyo, Japan, in 2007. From 2009 to 2010, Dr. Yang was a Postdoctoral Researcher in the department of electrical and computer engineering at the University of Utah, Salt Lake City, where he worked on electrochemical biosensors. He is currently a senior engineer at LG Chemical Development Corp., Daejeon, Korea. His research interests include biosensors, nano-particle functionalizations, MEMS process, microfabrication of carbon materials, enzymatic bio-fuel cell, electrochemistry, and surface chemistry.

Hyun Tae Kim received the B.E. degree in mechanical engineering from Korea University, Seoul, Korea, in 2006, and the M.S. degree in electrical engineering from the University of Utah, Salt Lake City, in 2012. He is currently working toward the Ph.D. degree in mechanical engineering at the University of Maryland, College Park. His research interests include micro sensors and actuators, bioMEMS, and medical robotics.

Hanseup Kim currently holds a position as an USTAR Assistant Professor of Electrical and Computer Engineering, of Mechanical Engineering, and of BioEngineering at the University of Utah in Salt Lake City, Utah since Fall 2009. He received his BS degree in Electrical Engineering from Seoul National University in 1997, and his MS and Ph.D. degrees in Electrical Engineering from the University of Michigan in 2002 and 2006, respectively. Between 2006 and 2009, he remained as a post-doctoral research fellow at the Center for Wireless Integrated MicroSystems (WIMS) in the University of Michigan. His research interests include: micro/nanofabrication technologies and structures, micro sensors and actuators, micropackaging, microfluidics, and bioMEMS.

Prof. Kim was awarded a National Science Foundation Faculty Early CAREER Award 2012 and a DARPA Young Faculty Award in 2011. He received the Best Paper Award with eight other co-authors from the International Conference on Commercialization of Micro and Nano Systems in 2008, the First Prize and the Best Paper Award with three other co-authors from the $38^{\text {th }}$ International Design Automation Conference in 2001, and Rotary Club Ambassador Scholarship in 1999. He has been active in the field of solid state sensors, actuators and microsystems, and has been served as a Technical Program Committee member for MEMS 2013, PowerMEMS 2012, the Hilton Head Workshop 2012, and NanoUtah Conferences.

\section{Acknowledgements}

This work was supported by the Utah Science Technology and Research (USTAR) initiative program. Microfabrication was performed at the Utah NanoFabrication Cleanroom Facility. The authors thank Dr. Michael Free and Mr. Prashant Saraswat in Metallurgical Engineering at the University of Utah for their help on the electrochemical measurement utilizing a potentiostat.

Received: 12 February 2014 Accepted: 15 April 2014

Published online: 07 June 2014

\section{References}

1. Alzheimer's Association (2012) Alz.org, Alzheimer's fact sheet. Available from: http://www.alz.org/documents_custom/2012_facts_figures_fact_sheet.pdf

2. Parkinson's Disease Foundation (2010) Parkinson's fact sheet. Available from: http://www.pdf.org/pdf/fs_frequently_asked_questions_10.pdf

3. National Institute of Neurological Disorders and Stroke. ALS(Amyotrophic Lateral Sclerosis) fact sheet, Huntington's disease: hope through research. Available from: http://www.ninds.nih.gov/disorders/huntington/detail_ huntington.htm

4. Van Den Eeden SK, Tanner CM, Bernstein AL, Fross RD, Leimpeter A, Bloch DA, Nelson LM (2002) Incidence of Parkinson's disease: variation by age, gender, and race/ethnicity. Am J Epidemiol 157:1015-1022

5. De Lau LML, Breteler MMB (2006) Epidemiology of Parkinson's disease. Lancet Neurol 5:525-535

6. Administration on Aging Older population by age group: 1900-2050 with persons +65. Available from: http:/www.aoa.gov/AoARoot/Aging_Statistics/ future_growth/future_growth.aspx\#age

7. Dauer W, Przedborski S (2003) Parkinson's disease: mechanisms and models. Neuron 39:889-909

8. Arias-Carrion O, Poppel E (2007) Dopamine, learning, and reward-seeking behavior. Act Neurobiol Exp 67:481-488

9. Shulman JM, De Jager PL, Feany MB (2011) Parkinson's disease: genetics and pathogenesis. Annu Rev Pathol Mech Dis 6:193-222

10. Zhao H, Zhang Y, Yuan Z (2001) Study on the electrochemical behavior of dopamine with poly (sulfosalicylic acid) modified glassy carbon electrode. Anal Chim Acta 441:117-122

11. Hjemdahl P (1984) Catecholamine measurements by high-performance liquid chromatography. Am J Physiol 247:E13-E20 
12. Bouchta D, Izaoumen N, Zejli H, Kaoutit ME, Temsamani KR (2005) A novel electrochemical synthesis of poly-3-methylthiophene-y-cyclodextrin film: Application for the analysis of chlorpromazine and some neurotransmitters. Biosen Bioelectron 20:2228-2235

13. Majewska UE, Chmurski K, Biesiada K, Olszyna AR, Bilewicz R (2006) Dopamine oxidation at per (6--deoxy--6--thio)--a--cyclodextrin monolayer modified gold electrodes. Electroanalysis 18:1463-1470

14. Alarcón-Angeles G, Pérez-López B, Palomar-Pardave M, Ramírez-Silva MT, Alegret S, Merkoçi A (2008) Enhanced host-guest electrochemical recognition of dopamine using cyclodextrin in the presence of carbon nanotubes. Carbon 46:898-906

15. Yang J-H, Park JW, Kim H (2010) Simultaneous detection of catecholamine neurotransmitters utilizing a cyclodextrin-based micro electrode array. MicroTAS 2010:599-601

16. Yang J-H, Kim H-T, Park JW, Kim H (2011) Cyclodextrin-based micro neurotransmitter sensor for selective catecholamine hormone detection. Transducers 2011:2102-2105

17. Tang H, Lin P, Chan HLW, Yan F (2011) Highly sensitive dopamine biosensors based on organic electrochemical transistors. Biosen Bioelectron 26:4559-4563

18. Pagel P, Blome J, Wolf HU (2000) High-performance liquid chromatographic separation and measurement of various biogenic compounds possibly involved in the pathomechanism of Parkinson's disease. J Chromatogr B 746:297-304

19. Wood AT, Hall MR (2000) Reversed-phase high-performance liquid chromatography of catecholamines and indoleamines using a simple gradient solvent system and native fluorescence detection. J Chromatogr B 744:221-225

20. Wang HY, Sun Y, Tang B (2002) Study on fluorescence property of dopamine and determination of dopamine by fluorimetry. Talanta 57:899-907

21. Balaji J, Reddy CS, Kaushalya SK, Maiti S (2004) Microfluorometric detection of catecholamines with multiphoton-excited fluorescence. Appl Optics 43:2412-2417

22. Tsunoda M, Takezawa K, Santa T, Imai K (1999) Simultaneous automatic determination of catecholamines and their 3-o-methyl metabolites in rat plasma by high-performance liquid chromatography using peroxyoxalate chemiluminescence reaction. Anal Biochem 269:386-392

23. Ragab GH, Nohta H, Zaitsu K (2000) Chemiluminescence determination of catecholamines in human blood plasma using 1,2-bis(3-chlorophenyl) ethylenediamine as pre-column derivatizing reagent for liquid chromatography Anal Chim Acta 403:155-160

24. Nalewajko E, Wiszowata A, Kojli A (2007) Determination of catecholamines by flow-injection analysis and high-performance liquid chromatography with chemiluminescence detection. J Pharm Biomed Anal 43:1673-1681

25. Hows MEP, Lacroix L, Heidbreder C, Organ AJ, Shah AJ (2004) High-performance liquid chromatography/tandem mass spectrometric assay for the simultaneous measurement of dopamine, norepinephrine, 5-hydroxytryptamine and cocaine in biological samples. J Neurosci Methods 138:123-132

26. Tsunoda M (2006) Recent advances in methods for the analysis of catecholamines and their metabolites. Anal Bioanal Chem 386:506-514

27. Ferancova A, Korgova E, Labuda J, Zima J, Barek J (2002) Cyclodextrin modified carbon paste based electrodes as sensors for the determination of carcinogenic polycyclic aromatic amines. Electroanalysis 23:1668-1673

28. Bouzitoun M, Mlika R, Gam H, Ouada HB, Majdoub M, Sfihi H (2006) A non-water-soluble modified $\beta$-cyclodextrin for sensitive electrode. Mater Sci Eng C 26:481-485

29. Li W, Jin G, Chen H, Kong J (2009) Highly sensitive and reproducible cyclodextrin-modified gold electrodes for probing trace lead in blood. Talanta 78:717-722

30. Tredici I, Merli D, Zavarise F, Profumo A (2010) a-Cyclodextrins chemically modified gold electrode for the determination of nitroaromatic compounds. J Electroanal Chem 645:22-27

31. Fukuda T, Maeda Y, Kitano H (1999) Stereoselective inclusion of DOPA derivatives by a self-assembled monolayer of thiolated cyclodextrin on a gold electrode. Langmuir 15:1887-1890
32. Fragoso A, Almirall E, Cao R, Echegoyen L, González-Jonte R (2004) A supramolecular approach to the selective detection of dopamine in the presence of ascorbate. Chem Commun 19:2230-2231

33. Palomar-Pardavé M, Alarcón-Ángeles G, Ramírez-Silva MT (2011) Electrochemical and spectrophotometric determination of the formation constants of the ascorbic acid- $\beta$-cyclodextrin and dopamine- $\beta$-cyclodextrin inclusion complexes. J Incl Phenom Macrocycl Chem 69:91-99

doi:10.1186/s40486-014-0001-z

Cite this article as: Yang et al:: A cyclodextrin-based approach for selective detection of catecholamine hormone mixtures. Micro and Nano Systems Letters 2014 2:1

\section{Submit your manuscript to a SpringerOpen ${ }^{\odot}$ journal and benefit from:}

- Convenient online submission

- Rigorous peer review

- Immediate publication on acceptance

- Open access: articles freely available online

- High visibility within the field

- Retaining the copyright to your article

Submit your next manuscript at $>$ springeropen.com 